\title{
Stridor Due to an Unsuspected Parathyroid Cyst in a Thirty-Two-Year-Old Woman with Goiter
}

\author{
Sima Saberi, ${ }^{1}$ Gerard M. Doherty, ${ }^{2}$ and Nazanene H. Esfandiari ${ }^{3}$
}

\section{Dear Editor:}

Parathyroid cysts are rare and can be difficult to diagnose, as they can be mistaken for thyroid cysts. Parathyroid cysts are of clinical relevance, as they can cause dysphagia, odynophagia, recurrent laryngeal nerve palsy, and hyperparathyroidism.

Here, we report an unusual young patient who developed stridor due to expansion of a large right parathyroid cyst that was initially thought to be a thyroid cyst. A 32-year-old woman with no significant past medical history was found to have a goiter on physical examination during evaluation of infertility. Her thyrotropin and calcium were normal. She had a thyroid ultrasound elsewhere and was found to have cystic lesions that were aspirated. Approximately 10 months later, she underwent a thyroid ultrasound at this institution that demonstrated a $6.3 \times 4.1 \times 2.5 \mathrm{~cm}$ cystic lesion in the mid to inferior aspect of the right thyroid lobe and a $2.5 \times 1.1 \times 1 \mathrm{~cm}$ cystic lesion in the mid to inferior aspect of the left thyroid lobe. The right cyst was aspirated yielding $55 \mathrm{~mL}$ of clear fluid, macrophages, and no thyroid follicular cells. The left lesion was biopsied and demonstrated abundant colloid without follicular cells. The patient declined to have a recommended thyroidectomy. Therefore, ultrasound was repeated 4 months later when she was asymptomatic. This showed recurrence of the right cyst, now $6.9 \times 4.1 \times 3.8 \mathrm{~cm}$ and a $2.5 \times 1.0 \times 0.8 \mathrm{~cm}$ complex cystic nodule in the posterior aspect of the mid to inferior left thyroid pole.

A month later, the patient came to the emergency department with stridor. She had both inspiratory and expiratory stridor and was placed on oxygen. Neck ultrasound showed an $\sim 4 \times 8 \mathrm{~cm}$ right cystic lesion. One hundred milliliters clear fluid was aspirated with relief of stridor; cytopathology showed rare follicular cells. She underwent a right thyroid lobectomy. Intraoperatively, a posteriorly extending cyst that was densely adherent to the trachea was noted. The cyst was resected after aspiration of $15 \mathrm{~mL}$ clear colorless fluid. Histopathology showed a parathyroid cyst and normal thyroid tissue (Supplementary Fig. 1; Supplementary Data are available online at www.liebertonline.com/thy).

Parathyroid microcysts are common at autopsy (1), but clinically symptomatic cysts are rare. Functional parathyroid cysts account for $\sim 1 \%$ of cases of primary hyperparathyroidism (2). The larger parathyroid cysts typically present as an asymptomatic neck mass in 40-60-year-old patients. Cysts can be as large as $15 \mathrm{~cm} \mathrm{(3)} \mathrm{and} \mathrm{found} \mathrm{from} \mathrm{the} \mathrm{mandible} \mathrm{to}$ the mediastinum (4). Differential diagnosis includes a thyroid cyst, thymus cyst, branchial cleft cyst, parathyroid carcinoma, or thyroglossal duct cyst (1). Parathyroid cysts commonly yield clear colorless fluid with an elevated parathyroid hormone level (4). The fluid in thyroid cysts is often blood tinged or brown with high thyroglobulin content. Parathyroid cysts may recur after aspiration (4), in which case surgical excision is recommended.

Rarely, patients with parathyroid cysts can present with symptoms of airway compression such as stridor. Stridor is a result of chaotic airflow through a partially occluded upper airway. Extrathoracic tracheal obstruction usually results in biphasic stridor (5). This was the likely cause of stridor in our patient, as the enlarging right parathyroid cyst was noted to be compressing her extrathoracic trachea.

This unusual case of a young woman with a recurrent parathyroid cyst causing stridor demonstrates the diagnostic and therapeutic challenge of parathyroid cysts and their clinically significant manifestations. Preoperatively, the possibility of a parathyroid cyst was not entertained in this patient, as her second aspiration had yielded rare follicular cells. In retrospect, it seems likely that the rare thyroid follicular cells in the cyst aspirate were due to fine needle aspiration outside of the cyst. The clear colorless fluid should have aroused our suspicion of a possible parathyroid cyst rather than a thyroid cyst and obtaining a parathyroid (PTH) level in the cyst aspirate, which was not done, would have been diagnostic. Clinicians should have a high index of suspicion for parathyroid cysts when clear colorless fluid is removed from anterior neck lesion.

\section{Acknowledgment}

The authors would like to thank Dr. Bryan Coffing for providing pathology images.

\section{References}

1. Alvi A, Myssiorek D, Wasserman P 1996 Parathyroid cyst: current diagnostic and management principles. Head and Neck 18:370-373.

2. Gough IR 1999 Parathyroid cysts. Aust N Z J Surg 69: 404-406.

\footnotetext{
${ }^{1}$ Ann Arbor Endocrinology and Diabetes Associates, PC, Ypsilanti, Michigan.

Divisions of ${ }^{2}$ Endocrine Surgery and ${ }^{3}$ Metabolism, Endocrinology, and Diabetes, University of Michigan, Ann Arbor, Michigan.
} 
3. Cao H, Lai CK, Head CS, Sercarz JA 2008 Cystic parathyroid presenting as an apparent thyroid goiter. Eur Arch Otorhinolaryngol 265:1285-1288.

4. Ujicki MB, Nayar R, Sturgeon C, Angelos P 2007 Parathyroid cyst: often mistaken for a thyroid cyst. World J Surg 31:60-64.

5. Majumdar S, Bateman NJ, Bull PD 2004 Pediatric Stridor. Arch Dis Child Educ Pract Ed 91:101-105.
Address correspondence to: Nazanene H. Esfandiari, M.D. Division of Metabolism, Endocrinology and Diabetes University of Michigan, Dominos Farms 24 Frank Lloyd Wright Drive Lobby C, Suite 1300 Ann Arbor, MI 48106

E-mail: nazanene@med.umich.edu 
This article has been cited by: 